Research Article

\title{
Finite Element Modeling for Static Bending Behaviors of Rotating FGM Porous Beams with Geometrical Imperfections Resting on Elastic Foundation and Subjected to Axial Compression
}

\author{
Nguyen Van Dang $\mathbb{D}$ \\ University of Transport Technology, 54 Trieu Khuc, Thanh Xuan, Hanoi, Vietnam \\ Correspondence should be addressed to Nguyen Van Dang; dangnv@utt.edu.vn
}

Received 10 April 2021; Accepted 24 November 2021; Published 10 December 2021

Academic Editor: Francisco Javier Fern ndez Fern ndez

Copyright (c) 2021 Nguyen Van Dang. This is an open access article distributed under the Creative Commons Attribution License, which permits unrestricted use, distribution, and reproduction in any medium, provided the original work is properly cited.

\begin{abstract}
The static bending analysis of the FG porous beam resting on the two-parameter elastic foundation is initially carried out using a combination of Reddy's high-order shear deformation theory and the finite element technique, where the initial geometrical imperfection and rotation movement in one fixed axis are calculated. Through the power-law distribution function with porosities, material characteristics vary constantly from one surface to the next in the direction of thickness, and the beam is concurrently impacted by an acting force perpendicular to the beam axis and an axial compressive force. The stiffness matrix of the beam element changes as a result, and the static bending response of this beam is significantly different from that of ordinary beams. Comparison cases with published findings are used to verify the computational theory. The calculations clearly reveal many innovations for rotating beams that are influenced by many different kinds of loads, which may be used to the designing, manufacturing, and usage of these structures in reality.
\end{abstract}

\section{Introduction}

With the advancement of science and technology, a vast array of novel materials has been created and used to manufacturing in vital sectors with high economic efficiency, such as military, high-speed rail, and nuclear technology, to name a few. Functionally graded materials (FGM) are one of the most popular novel materials in recent years. They are made up of two or more component elements, although the most frequent are ceramic and metal combined in certain proportions. As a result, FGM components offer all of the benefits of both ceramics and metals, including heat resistance, friction and abrasion resistance, and more strength than conventional steels. However, several flaws may emerge during the manufacturing process, the most frequent of which is the appearance of tiny porosities in the material. The presence of these microscopic porosities has a major impact on the efficiency of the structures' operating performance. As a consequence, mechanical professionals are also highly willing to investigate the mechanical behavior of structures constructed of FGM materials with porosities, and some significant research results have been obtained. To explore the nonlinear static deflections of functionally graded porosity under heat influence, Akbas [1] used the complete Lagrangian finite element technique within the two-dimensional continuum model in the Newton-Raphson method. According to Timoshenko beam theory, Mojahedin et al. [2] performed a thermoelastic study of functionally graded porous beams under in-plane thermal loading, which was applied as a uniform temperature distribution throughout the whole beam. Eltaher et al. [3] investigated the mechanical bending and vibration of functionally graded porous nanobeams using the Euler-Bernoulli and finite element methods (FEM). Hamed et al. [4] employed Euler-Bernoulli beam theory and the finite element method to model the mechanical bending behaviors of functionally graded porous nanobeams. Based on a novel improved quasi-3D shear deformation theory, Fahsi et al. [5] employed the Navier solution to investigate the bending, buckling, and free vibration responses of a 
functionally graded porous beam resting on an elastic basis. Based on a trigonometric shear deformation theory and finite element technique, Anirudha et al. [6] investigated the bending, vibration, and buckling properties of functionally graded porous graphene-reinforced nanocomposite curved beams. Polit et al. [7] utilized Navier's solutions and a higher-order shear deformation theory to investigate the static bending and elastic stability of thick functionally graded graphene platelets reinforced porous nanocomposite curved beams. Masjedi et al. [8] used an orthogonal Chebyshev collocation approach to investigate the large deflection behavior of functionally graded porous beams under conservative and nonconservative (follower) loading situations. Gao et al. [9] investigated the nonlinear bending of a functionally graded porous nanobeam exposed to various physical stresses using a two-step perturbation technique and the nonlocal strain gradient theory. Using a modified mixed finite element beam model, Zghal et al. [10] investigated the effect of porosity on bending static analysis of functionally graded beams. Hamed et al. [11] examined the mechanical responses of a thin/thick sandwich functionally graded beam with a porous core using a parabolic higherorder shear deformation theory. Using the extended differential quadrature approach, Nan et al. [12] developed the governing differential equations and boundary conditions of functionally gradient piezoelectric nanobeams with porosities. Based on Euler-Bernoulli beam theory, Timoshenko beam theory, and Reddy's third-order shear deformation theory, Enayat et al. [13] provided a size-dependent mechanical study of functionally graded porous nanobeams resting on Pasternak foundation in the thermal environment. Zhao et al. [14] investigated the static bending and free vibration of a size-dependent porous axially functional gradient flexoelectric Euler-Bernoulli nanobeam using the extended differential quadrature technique. Pajand et al. [15] proposed an economical and high-performance four-node isoparametric beam element for thermo-mechanical nonlinear analysis of functionally graded porous beams. Karamanli and Vo [16] used a finite element model based on a quasi-3D theory and the modified strain gradient theory to simulate the bending, vibration, and buckling behaviors of bidirectional FG porous microbeams.

Some mechanical parts may be involved in rotational motions in technological procedures, such as revolving railway bridges and rotor blades. As a result, mechanical behavior studies of these components with rotational movements are crucial in computational design, which has piqued the curiosity of experts all over the world. The following are some notable publications to consider. The findings of the mechanical reactions of spinning nanobeams utilizing the differential quadrature technique were presented by Pradhan and Murmu [17]. Based on a dynamic model, Li et al. [18] investigated the influence of bending and stretching on the free-vibration responses of a rotating FGM beam. Das [19] studied the in-plane and out-of-plane mechanical responses of rotating FGM beams using the Ritz technique and Timoshenko theory. Chen et al. [20] provided the findings of a vibration study of spinning microbeams that took into consideration the geometrical imperfection at the outset. In [21], the author also discussed the bending of beams rotating around a fixed axis, as well as the influence of temperature on FGM material without porosities. In this work, the author looks at materials having pore-type defects as well as the effect of compressive forces along the beam's axis. As a result, material objects and external loads acting on the structure are considerably different. Compared to the previous study [21], this will significantly modify the beam structure's response.

Based on the foregoing review, it is clear that no publications exists that deal with the bending analysis of rotating FG porous beams resting on an elastic foundation, in which the beam is simultaneously impacted by an axial compressive load and a force applied perpendicular to the beam axis. As a result, this work is a unique investigation that adds substantial value to engineering methods. As a result, the focus of this research is on the static bending behaviors of the aforementioned structures using Reddy's high-order shear deformation theory (HSDT) and the FEM.

The remainder of this work is structured in the following manner. The effects of the initial geometrical imperfection and the loads in both the thickness and longitudinal dimensions are examined in the finite element formulas for the bending issue of rotating porous FGM beams resting on elastic foundations in Section 2. In Section 3, there are examples of verification. The bending reactions of rotating porous FGM beams are investigated numerically and discussed in Section 4. Section 5 summarizes the novel aspects of this work.

\section{Finite Element Model of Rotating Porous FGM Beam}

The goal of this work is to compute a FG beam using the model illustrated in Figure 1. The structure is embedded in a two-parameter elastic foundation with $k_{w}$ and $k_{s}$ coefficients, and the beam has length $L$, breadth $b$, and thickness $h$. The beam is formed of FGM material with a pore-defect of the material with a porosity volume fraction $\alpha$ and has an initial geometric imperfection $w_{\text {imp }}(x)$ in the $z$-direction. The entire mechanical system rotates at a constant speed $\phi$ around one fixed axis $T$, one side of the beam is offset from the axis $r$, and the beam is compressed axially by the load $N_{0}$.

FG beams are often composed of two or more distinct materials with smooth material characteristics in one or two dimensions. This research focuses on beams consisting of two materials, ceramic and metal, which change depending on the thickness of the beam, with the volume of ceramic $\left(V_{c}\right)$ and metal $\left(V_{m}\right)$ stated as follows [22-28]:

$$
\begin{aligned}
V_{m}+V_{c} & =1, \\
V_{c} & =\left(\frac{z}{h}+\frac{1}{2}\right)^{n} \text { with } n \geq 0,
\end{aligned}
$$

where $n$ denotes the volume fraction gradient index and its fluctuation, $z$ is the thickness coordinate variable with $-h / 2 \leq z \leq h / 2$, and subscripts $c$ and $m$ denote the ceramic and metal components, respectively. 


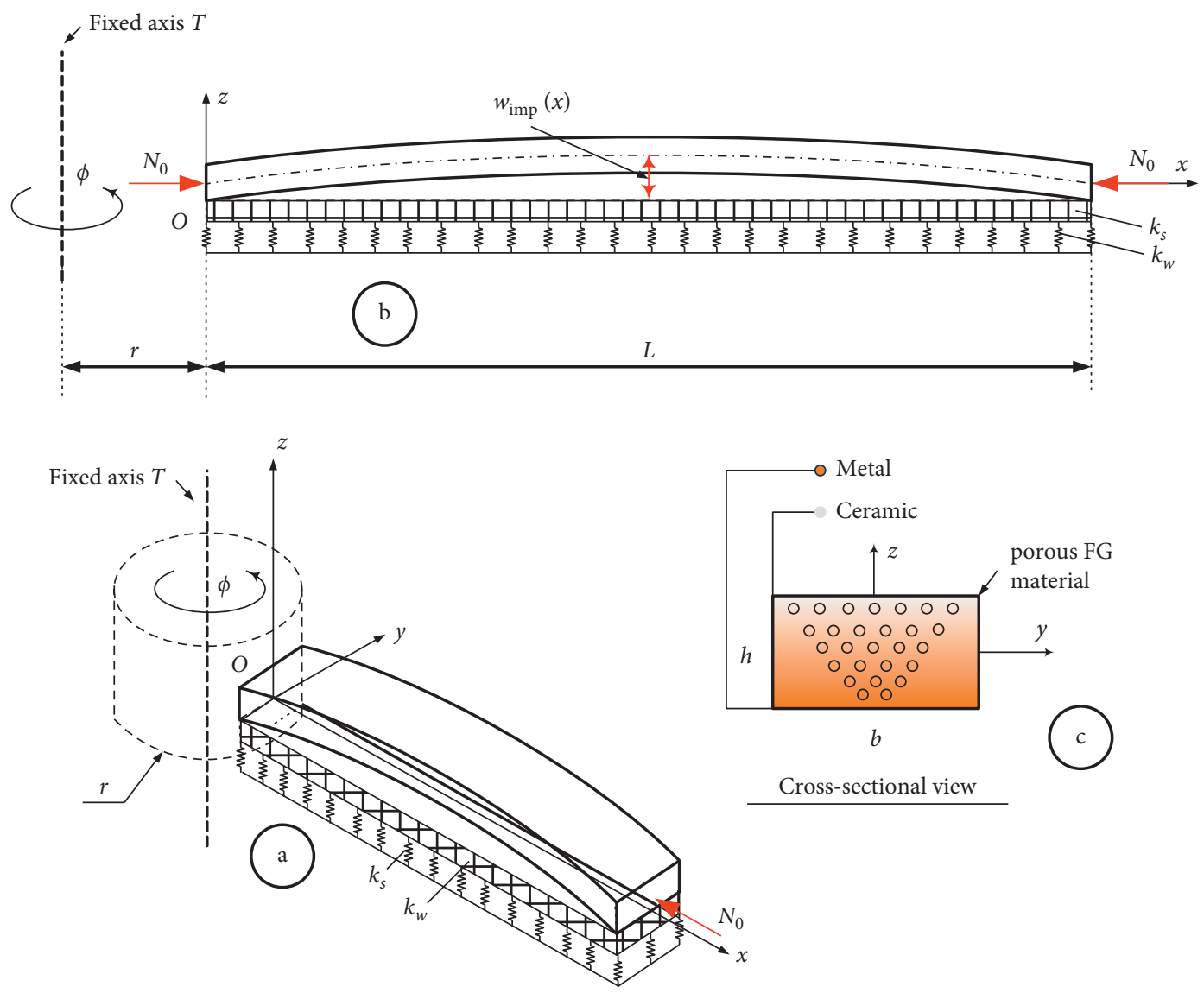

FIgURE 1: A rotating porous FGM beam resting on an elastic foundation (a) General perspective. (b) xOz perspective. (c) Cross-sectional perspective.

Based on the power-law distribution, material characteristics such as Young's modulus $E$, mass density $\rho$, and Poisson's ratio $v$ vary in the $z$-direction as [22-28]

$$
\begin{aligned}
& E(z)=E_{m}+\left(E_{c}-E_{m}\right)\left(\frac{1}{2}+\frac{z}{h}\right)^{n}-\frac{\alpha}{2}\left(E_{c}+E_{m}\right), \\
& \rho(z)=\rho_{m}+\left(\rho_{c}-\rho_{m}\right)\left(\frac{1}{2}+\frac{z}{h}\right)^{n}-\frac{\alpha}{2}\left(\rho_{c}+\rho_{m}\right), \\
& \nu(z)=v_{m}+\left(v_{c}-v_{m}\right)\left(\frac{1}{2}+\frac{z}{h}\right)^{n}-\frac{\alpha}{2}\left(v_{c}+v_{m}\right),
\end{aligned}
$$

where $\alpha$ is a porosity volume fraction $(\alpha<1)$.

Different beam theories, ranging from classical beam theory (CBT) to higher-order shear deformation theory, can be utilized to create computational equations for beam structures (HSDT). This study employs Reddy's third-order shear deformation theory, which necessitates more complex calculation formulas and takes longer to calculate than lower-order theories. This theory, on the contrary, does not require a shear correction factor since it fulfills the zerostress boundary condition on the upper and lower surfaces of the beam, while still properly describing the structure's mechanical response. For thin to thick beams, especially thick beams, Reddy's third-order shear deformation theory can be used, which more precisely describes the mechanical reaction than classical theories and the first-order shear deformation theory.
The displacement field has the following expression [29] at any location $(x, z)$ within the beam:

$$
\left\{\begin{array}{l}
u(x, z)=u_{0}(x, 0)+z \varphi_{x}(x, y)-\frac{4}{3 h^{2}} z^{3}\left(\varphi_{x}+\frac{\partial w_{0}}{\partial x}\right), \\
w(x, z)=w_{0}(x, 0)+w_{\mathrm{imp}}(x) .
\end{array}\right.
$$

where $u_{0}(x, 0)$ and $w_{0}(x, 0)$ are the displacements of the point with the $x$ coordinate in the neutral axis, respectively, whereas $\varphi_{x}$ indicates the $y$-axis transverse normal rotation.

Individual derivatives are used to compute the longitudinal and shear strain components as follows:

$$
\begin{aligned}
\varepsilon_{x x} & =\frac{\partial u}{\partial x}=\frac{\partial u_{0}}{\partial x}+z \frac{\partial \varphi_{x}}{\partial x}+\frac{-4 z^{3}}{3 h^{2}}\left(\frac{\partial \varphi_{x}}{\partial x}+\frac{\partial^{2} w_{0}}{\partial x^{2}}\right)+\frac{\partial w_{0}}{\partial x} \frac{\mathrm{d} w_{\text {imp }}}{\mathrm{d} x} \\
& =\varepsilon_{0 x}+z \varepsilon_{1 x}+z^{3} \varepsilon_{3 x}+\varepsilon_{\mathrm{imp}}, \\
\gamma_{x z} & =\varphi_{x}+\frac{\partial w_{0}}{\partial x}+z^{2} \frac{-4}{h^{2}}\left(\varphi_{x}+\frac{\partial w_{0}}{\partial x}\right) \\
& =\gamma_{0 x z}+z^{2} \gamma_{2 x z},
\end{aligned}
$$


where $w_{\text {imp }}$ represents the beam's initial geometrical imperfection (see Figure 1) and $\varepsilon_{\text {imp }}$ represents the strain component caused by the first geometrical imperfection.

The following is how Hook's law is used to determine stress components:

$$
\left\{\begin{array}{l}
\sigma_{x x}=E \varepsilon_{x x}, \\
\tau_{x z}=\frac{E}{2(1+\nu)} \gamma_{x z} .
\end{array}\right.
$$

A weak form of the static bending model for FGM beams is as follows:

$$
\delta\left(U^{E}+U^{F}+U^{R}-U^{\text {Axi }}\right)-\delta W^{\text {For }}=0 .
$$

In this case, the weak form of elastic energy is represented as follows:

$$
\begin{aligned}
& \delta U^{E}=\int_{V}\left(\delta \sigma_{x x}^{T} \varepsilon_{x x}+\delta \tau_{x z}^{T} \gamma_{x z}\right) \mathrm{d} V \\
& =\int_{V}\left(\delta\left\{\begin{array}{llll}
\varepsilon_{0 x} & z \varepsilon_{1 x} & z^{3} \varepsilon_{3 x} & \varepsilon_{\text {imp }}
\end{array}\right\}^{T} E\left\{\begin{array}{c}
\varepsilon_{0 x} \\
z \varepsilon_{1 x} \\
z^{3} \varepsilon_{3 x} \\
\varepsilon_{\text {imp }}
\end{array}\right\}\right) \mathrm{d} V+\int_{V} \frac{E}{2(1+\nu)}\left(\delta\left\{\gamma_{0 x z} z^{2} \gamma_{2 x z}\right\}^{T}\left\{\begin{array}{c}
\gamma_{0 x z} \\
z^{2} \gamma_{2 x z}
\end{array}\right\}\right) \mathrm{d} V \\
& =\int_{V}\left(\begin{array}{c}
\delta \varepsilon_{0 x}^{T} E \varepsilon_{0 x}+\delta \varepsilon_{0 x}^{T} z E \varepsilon_{1 x}+\delta \varepsilon_{0 x}^{T} z^{3} E \varepsilon_{3 x}+\delta \varepsilon_{0 x}^{T} E \varepsilon_{\text {imp }} \\
\delta \varepsilon_{1 x}^{T} E z \varepsilon_{0 x}+\delta \varepsilon_{1 x}^{T} E z^{2} \varepsilon_{1 x}+\delta \varepsilon_{1 x}^{T} E z^{4} \varepsilon_{3 x}+\delta \varepsilon_{1 x}^{T} E z \varepsilon_{\text {imp }} \\
\delta \varepsilon_{3 x}^{T} E z^{3} \varepsilon_{0 x}+\delta \varepsilon_{3 x}^{T} E z^{4} \varepsilon_{1 x}+\delta \varepsilon_{3 x}^{T} E z^{6} \varepsilon_{3 x}+\delta \varepsilon_{3 x}^{T} E z^{3} \varepsilon_{\text {imp }} \\
\delta \varepsilon_{\text {imp }}^{T} E \varepsilon_{0 x}+\delta \varepsilon_{\text {imp }}^{T} E z \varepsilon_{1 x}+\delta \varepsilon_{\text {imp }}^{T} E z^{3} \varepsilon_{3 x}+\delta \varepsilon_{\text {imp }}^{T} E \varepsilon_{\text {imp }}
\end{array}\right) d V+\int_{V}\left(\begin{array}{c}
\delta \gamma_{0 x z}^{T} \frac{E}{2(1+\nu)} \gamma_{0 x z}+\delta \gamma_{0 x z}^{T} \frac{E z^{2}}{2(1+\nu)} \gamma_{2 x z} \\
+\delta \gamma_{2 x z}^{T} \frac{E z^{2}}{2(1+\nu)} \gamma_{0 x z}+\delta \gamma_{2 x z}^{T} \frac{E z^{4}}{2(1+\nu)} \gamma_{2 x z}
\end{array}\right) \mathrm{d} V
\end{aligned}
$$

The weak form of elastic energy is expressed as follows in this case:

$$
\delta U^{F}=b \int_{L}\left(k_{w} \delta w_{0} w_{0}+k_{s} \delta\left(\frac{\partial w_{0}}{\partial x}\right)\left(\frac{\partial w_{0}}{\partial x}\right)\right) \mathrm{d} x,
$$

where $k_{w}$ and $k_{s}$ are the elastic foundation's two coefficients.

The weak form of the potential energy generated by the rotational movement of the FGM beam spinning around one axis $\Delta$ at the speed $\phi$ is computed as $[20,30]$

$$
\delta U^{R}=\int_{L}\left(F_{\phi}(x) \delta\left(\frac{\partial w_{0}}{\partial x}\right)\left(\frac{\partial w_{0}}{\partial x}\right)\right) \mathrm{d} x,
$$

in which the centrifugal force $F_{\phi}[20]$ is

$$
F_{\phi}=\frac{1}{2} \int_{S}\left(\rho \phi^{2}\left[r(L-x)+\frac{1}{2}\left(L^{2}-x^{2}\right)\right]\right) \mathrm{d} S,
$$

where $\rho$ is the density of the material.

The axial compressive load acting on the beam's weak form is computed as [30]

$$
\delta U^{\mathrm{Axi}}=\int_{L}\left(N_{0} \delta\left(\frac{\partial w_{0}}{\partial x}\right)\left(\frac{\partial w_{0}}{\partial x}\right)\right) \mathrm{d} x,
$$

where $N_{0}$ denotes the beam's compressive load (see Figure 1).

The external evenly distributed load $F_{0}$ acting on the FGM beam causes the weak form, which is computed as

$$
\delta W^{\text {For }}=b . h \cdot \int_{L}\left(\delta w_{0}^{T} \mathbf{F}_{0}\right) \mathrm{d} x,
$$

where $b$ and $h$ are the beam's width and thickness, respectively (see Figure 1).

To create the beam's equilibrium in the finite element formula, the beam must be divided into finite elements; this work uses the two-node beam element, with each node having four degrees of freedom:

$$
\mathbf{q}_{e}=\sum_{i=1}^{2}\left\{\begin{array}{c}
u_{0 i} \\
\varphi_{x i} \\
w_{0 i} \\
\left(\frac{\partial w_{0}}{\partial x}\right)_{i}
\end{array}\right\} .
$$


As a result, each displacement component is approximated as follows using the Lagrange $\left(N_{i}\right)$ and Hermit $\left(H_{i}\right)$ shape functions and the node displacement vector $\boldsymbol{q}_{e}$ :

$$
\left\{\begin{array}{l}
u_{0}=\sum_{i=1}^{2} N_{i} u_{0 i}=\mathbf{N}_{u} \mathbf{q}_{e}, \\
\varphi_{x}=\sum_{i=1}^{2} N_{i} \varphi_{x i}=\mathbf{N}_{\varphi} \mathbf{q}_{e}, \\
\frac{w_{0}=\sum_{i=1}^{2}\left\{H_{i} w_{0 i}+H_{i+1}\left(\frac{\partial w_{0}}{\partial x}\right)_{i}\right\}=\mathbf{H q}_{e},}{\partial x}=\sum_{i=1}^{2}\left\{\frac{\partial H_{i}}{\partial x} w_{0 i}+\frac{\partial H_{i+1}}{\partial x}\left(\frac{\partial w_{0}}{\partial x}\right)_{i}\right\}=\mathbf{H}_{1} \mathbf{q}_{e}, \\
\frac{\partial^{2} w_{0}}{\partial x^{2}}=\sum_{i=1}^{2}\left\{\frac{\partial^{2} H_{i}}{\partial x^{2}} w_{0 i}+\frac{\partial^{2} H_{i+1}}{\partial x^{2}}\left(\frac{\partial w_{0}}{\partial x}\right)_{i}\right\}=\mathbf{H}_{2} \mathbf{q}_{e} .
\end{array}\right.
$$

Equation (14) may be rewritten in a more concise manner as $\mathbf{u}=\left\{\begin{array}{c}u_{0} \\ \varphi_{x} \\ w_{0} \\ \left(\frac{\partial w_{0}}{\partial x}\right)\end{array}\right\}$<smiles>C[C@@H]1[CH]C=NC1</smiles>$$
=\mathbf{H}_{q} \cdot \mathbf{q}_{e} \text {. }
$$

When strain components are represented using the nodal displacement vector, the following results are obtained:

$$
\begin{aligned}
& \left\{\begin{array}{l}
\varepsilon_{0 x}=\frac{\partial u_{0}}{\partial x}=\frac{\partial \mathbf{N}_{u}}{\partial x} \mathbf{q}_{e}=\mathbf{B}_{0 x} \mathbf{q}_{e} \\
\varepsilon_{1 x}=\frac{\partial \varphi_{x}}{\partial x}=\frac{\partial \mathbf{N}_{\varphi}}{\partial x} \mathbf{q}_{e}=\mathbf{B}_{1 x} \mathbf{q}_{e} \\
\varepsilon_{3 x}=\frac{-4 z^{3}}{3 h^{2}}\left(\frac{\partial \varphi_{x}}{\partial x}+\frac{\partial^{2} w_{0}}{\partial x^{2}}\right)=\frac{-4 z^{3}}{3 h^{2}}\left(\frac{\partial \mathbf{N}_{\varphi}}{\partial x}+\mathbf{H}_{2}\right) \mathbf{q}_{e}=\mathbf{B}_{3 x} \mathbf{q}_{e} \\
\varepsilon_{\text {imp }}=\frac{\partial w_{0}}{\partial x} \frac{\mathrm{d} w_{\mathrm{imp}}}{\mathrm{d} x}=\frac{\mathrm{d} w_{\mathrm{imp}}}{\mathrm{d} x} \mathbf{H}_{1} \mathbf{q}_{e}=\mathbf{B}_{\mathrm{imp}} \mathbf{q}_{e} \\
\gamma_{0 x z}=\varphi_{x}+\frac{\partial w_{0}}{\partial x}=\mathbf{N}_{\varphi} \mathbf{q}_{e}+\mathbf{H}_{1} \mathbf{q}_{e}=\left(\mathbf{N}_{\varphi}+\mathbf{H}_{1}\right) q_{e}=\mathbf{B}_{0 \gamma} \mathbf{q}_{e} \\
\gamma_{2 x z}=\frac{-4}{h^{2}}\left(\varphi_{x}+\frac{\partial w_{0}}{\partial x}\right)=\frac{-4}{h^{2}}\left(\mathbf{N}_{\varphi} \mathbf{q}_{e}+\mathbf{H}_{1} \mathbf{q}_{e}\right)=\frac{-4}{h^{2}}\left(\mathbf{N}_{\varphi}+\mathbf{H}_{1}\right) \mathbf{q}_{e}=\mathbf{B}_{2 \gamma} \mathbf{q}_{e}
\end{array}\right.
\end{aligned}
$$

As a result, the element FGM beam's weak form of elastic energy is represented as follows: 


$$
\begin{aligned}
& \delta U_{e}^{E}=\delta \mathbf{q}_{e}^{T} \int_{V}\left(\begin{array}{c}
\mathbf{B}_{0 x}^{T} E \mathbf{B}_{0 x}+\mathbf{B}_{0 x}^{T} z E \mathbf{B}_{1 x}+\mathbf{B}_{0 x}^{T} z^{3} E \mathbf{B}_{3 x}+\mathbf{B}_{0 x}^{T} E z \mathbf{B}_{\mathrm{imp}} \\
\mathbf{B}_{3 x}^{T} E z^{3} \mathbf{B}_{0 x}+\mathbf{B}_{1 x}^{T} E z^{2} \mathbf{B}_{1 x}+\mathbf{B}_{1 x}^{T} E z^{4} \mathbf{B}_{3 x}+\mathbf{B}_{1 x}^{T} E z \mathbf{B}_{1 x}+\mathbf{B}_{3 x}^{T} E z^{6} \mathbf{B}_{3 x}+\mathbf{B}_{3 x}^{T} E z^{3} \mathbf{B}_{\mathrm{imp}} \\
\mathbf{B}_{\mathrm{imp}}^{T} E \mathbf{B}_{0 x}+\mathbf{B}_{\mathrm{imp}}^{T} E z \mathbf{B}_{1 x}+\mathbf{B}_{\mathrm{imp}}^{T} E z^{3} \mathbf{B}_{3 x}+\mathbf{B}_{\mathrm{imp}}^{T} E \mathbf{B}_{\mathrm{imp}}
\end{array}\right) \mathrm{d} V \mathrm{q}_{e} \\
& +\delta \mathbf{q}_{e}^{T} \int_{V}\left(\mathbf{B}_{0 x z}^{T} \frac{E}{2(1+\nu)} \mathbf{B}_{0 x z}+\mathbf{B}_{0 x z}^{T} \frac{E z^{2}}{2(1+\nu)} B_{2 x z}+\mathbf{B}_{2 x z}^{T} \frac{E z^{2}}{2(1+\nu)} \mathbf{B}_{0 x z}+\mathbf{B}_{2 x z}^{T} \frac{E z^{4}}{2(1+\nu)} \mathbf{B}_{2 x z}\right) \mathrm{d} V \mathbf{q}_{e} \\
& =\delta \mathrm{q}_{e}^{T} \int_{V}\left(\begin{array}{c}
\mathbf{B}_{0 x}^{T} E \mathbf{B}_{0 x}+\mathbf{B}_{0 x}^{T} z E \mathbf{B}_{1 x}+\mathbf{B}_{0 x}^{T} z^{3} E \mathbf{B}_{3 x}+\mathbf{B}_{0 x}^{T} E \mathbf{B}_{\mathrm{imp}} \\
\mathbf{B}_{3 x}^{T} E z \mathbf{B}_{0 x}+\mathbf{B}_{1 x}^{T} E z^{2} \mathbf{B}_{1 x}+\mathbf{B}_{1 x}^{T} E z^{4} \mathbf{B}_{3 x}+\mathbf{B}_{1 x}^{T} E z \mathbf{B}_{\mathrm{imp}}^{T} E z^{4} \mathbf{B}_{1 x}+\mathbf{B}_{3 x}^{T} E z^{6} \mathbf{B}_{3 x}+\mathbf{B}_{3 x}^{T} E z^{3} \mathbf{B}_{\mathrm{imp}} \\
\mathbf{B}_{\mathrm{imp}}^{T} E \mathbf{B}_{0 x}+\mathbf{B}_{\mathrm{imp}}^{T} E z \mathbf{B}_{1 x}+\mathbf{B}_{\mathrm{imp}}^{T} E z^{3} \mathbf{B}_{3 x}+\mathbf{B}_{\mathrm{imp}}^{T} E \mathbf{B}_{\mathrm{imp}}
\end{array}\right) \mathrm{d} V \mathbf{q}_{e} \\
& +\delta \mathbf{q}_{e}^{T} \int_{V}\left(\mathbf{B}_{0 x z}^{T} \frac{E}{2(1+\nu)} \mathbf{B}_{0 x z}+\mathbf{B}_{0 x z}^{T} \frac{E z^{2}}{2(1+\nu)} \mathbf{B}_{2 x z}+\mathbf{B}_{2 x z}^{T} \frac{E z^{2}}{2(1+\nu)} \mathbf{B}_{0 x z}+\mathbf{B}_{2 x z}^{T} \frac{E z^{4}}{2(1+\nu)} \mathbf{B}_{2 x z}\right) \mathrm{d} V \mathbf{q}_{e} .
\end{aligned}
$$

In matrix form, the above equation is written as

$$
\delta U_{e}^{E}=\delta \mathbf{q}_{e}^{T} \mathbf{K}_{e}^{E} \mathbf{q}_{e},
$$

where the stiffness matrix of the elements is computed as follows:

$$
\begin{aligned}
& \mathbf{K}_{e}^{E}=\int_{V}\left(\begin{array}{c}
\mathbf{B}_{0 x}^{T} E \mathbf{B}_{0 x}+\mathbf{B}_{0 x}^{T} z E \mathbf{B}_{1 x}+\mathbf{B}_{0 x}^{T} z^{3} E \mathbf{B}_{3 x}+\mathbf{B}_{0 x}^{T} E \mathbf{B}_{\mathrm{imp}} \\
\mathbf{B}_{1 x}^{T} E z \mathbf{B}_{0 x}+\mathbf{B}_{1 x}^{T} E z^{2} \mathbf{B}_{1 x}+\mathbf{B}_{1 x}^{T} E z^{4} \mathbf{B}_{3 x}+\mathbf{B}_{1 x}^{T} E z \mathbf{B}_{\mathrm{imp}} \\
\mathbf{B}_{3 x}^{T} E z^{3} \mathbf{B}_{0 x}+\mathbf{B}_{3 x}^{T} E z^{4} \mathbf{B}_{1 x}+\mathbf{B}_{3 x}^{T} E z^{6} \mathrm{~B}_{3 x}+\mathrm{B}_{3 x}^{T} E z^{3} \mathrm{~B}_{\mathrm{imp}} \\
\mathbf{B}_{\mathrm{imp}}^{T} E \mathbf{B}_{0 x}+\mathbf{B}_{\mathrm{imp}}^{T} E z \mathbf{B}_{1 x}+\mathbf{B}_{\mathrm{imp}}^{T} E z^{3} \mathbf{B}_{3 x}+\mathbf{B}_{i m p}^{T} E \mathbf{B}_{\mathrm{imp}}
\end{array}\right) \mathrm{d} V \\
& +\int_{V}\left(\mathbf{B}_{0 x z}^{T} \frac{E}{2(1+\nu)} \mathbf{B}_{0 x z}+\mathbf{B}_{0 x z}^{T} \frac{E z^{2}}{2(1+\nu)} \mathbf{B}_{2 x z}+\mathbf{B}_{2 x z}^{T} \frac{E z^{2}}{2(1+\nu)} \mathbf{B}_{0 x z}+\mathbf{B}_{2 x z}^{T} \frac{E z^{4}}{2(1+\nu)} \mathbf{B}_{2 x z}\right) \mathrm{d} V . \\
& \mathbf{K}_{e}^{F}=b \int_{L}\left(k_{1} \mathbf{H}^{T} \mathbf{H}+k_{2} \mathbf{H}_{1}^{T} \mathbf{H}_{1}\right) \mathrm{d} x, \\
& \mathbf{K}_{e}^{R}=\int_{L}\left(F_{\phi}(x) \mathbf{H}_{1}^{T} \mathbf{H}_{1}\right) \mathrm{d} x, \\
& \mathbf{K}_{e}^{\mathrm{Axi}}=\int_{L}\left(N_{0} \mathbf{H}_{1}^{T} \mathbf{H}_{1}\right) \mathrm{d} x .
\end{aligned}
$$

The axial compressive load $N_{0}$ and the weak form of the elastic foundation, centrifugal inertia force, have the following expression:

$$
\begin{aligned}
\delta U_{e}^{F} & =\delta \mathbf{q}_{e}^{T}\left(b \int_{L}\left(k_{1} \mathbf{H}^{T} \mathbf{H}+k_{2} \mathbf{H}_{1}^{T} \mathbf{H}_{1}\right) \mathrm{d} x\right) \mathbf{q}_{e}=\delta \mathbf{q}_{e}^{T} \mathbf{K}_{e}^{F} \mathrm{q}_{e} \\
\delta U_{e}^{R} & =\delta \mathbf{q}_{e}\left(\int_{L}\left(F_{\phi}(x) \mathbf{H}_{1}^{T} \mathbf{H}_{1}\right) \mathrm{d} x\right) \mathbf{q}_{e}=\delta \mathbf{q}_{e}^{T} \mathbf{K}_{e}^{R} \mathbf{q}_{e} \\
\delta U^{A x i} & =\delta \mathbf{q}_{e}\left(\int_{L}\left(N_{0} \mathbf{H}_{1}^{T} \mathbf{H}_{1}\right) \mathrm{d} x\right) \mathbf{q}_{e}=\delta \mathbf{q}_{e}^{T} \mathbf{K}_{e}^{A x i} \mathbf{q}_{e}
\end{aligned}
$$

Finally, the work done by evenly distributed load $F_{0}$ operating on the FGM beam element is used to derive the force vector acting on the beam element:

$$
\begin{aligned}
\delta W_{e}^{\text {For }} & =\delta \mathbf{q}_{e}^{T}\left(b \int_{L}\left(\mathbf{H}^{T} \mathbf{F}_{0}\right) \mathrm{d} x\right) \\
& =\delta \mathbf{q}_{e}^{T} \mathbf{F}_{e},
\end{aligned}
$$

where 
where the element's nodal force vector is computed as

$$
\mathbf{F}_{e}=b \int_{L}\left(\mathbf{H}^{T} \mathbf{F}_{0}\right) \mathrm{d} x .
$$

The equation for the static bending of FG porous beams is defined by substituting equations (18), (20), and (22) into (6):

$$
\sum_{e}\left(\mathbf{K}_{e}^{E}+\mathbf{K}_{e}^{F}+\mathbf{K}_{e}^{R}-\mathbf{K}_{e}^{\mathrm{Axi}}\right) \mathbf{q}_{e}=\sum_{e} \mathbf{F}_{e}
$$

The beam element stiffness matrix is thus related to the rotational speed, elastic foundation coefficients $k_{\mathrm{w}}$ and $k_{\mathrm{s}}$, and geometrical imperfection coefficient $w_{0}$ of the FGM beam, which is completely different from conventional beams; as a result, the static bending response of this beam differs from published results. Furthermore, by constructing computational formulas utilizing third-order shear deformation theory, this study eliminates the requirement for a shear correction factor, which helps to correctly characterize the mechanical reactions of FG beam systems.

The boundary requirements for this calculation for beams are as follows:

(i) Two sides of the beam are simply supported (denoted as S-S); each side meets the following constraints:

$$
u_{0}=0, \quad w=0
$$

(ii) The beam is clamped on two sides (designated C-C), with each side meeting the following requirements:

$$
\begin{gathered}
u_{0}=0, \\
\varphi_{x}=0, \\
w=0, \\
\frac{\partial w}{\partial x}=0 .
\end{gathered}
$$

\section{Examples of Verification}

This section compares the static deflections of the FG beams predicted by this study with those calculated by other methods to validate the reliability of the computational theory proposed in Section 2.

Example 1. The static deflections of the FGM porous beam under an evenly distributed static load are compared in this example. $E_{m}=70 \mathrm{GPa}, E_{c}=380 \mathrm{GPa}$, and $\nu_{c}=v_{m}=0.3$ are the material characteristics of the beam, which are fully simply supported. For this problem, the top surface of the beam is metal and the bottom surface is ceramic; the uniformly distributed static load is $P_{0}=10^{6} \mathrm{~N} / \mathrm{m}^{2}$, the length is $L$, and the thickness $h=L / 5$. The formula normalizes the nondimensional maximum deflection, $\bar{w}=100 E_{m} h^{3} / P_{0} L^{4} w_{\max }$. Table 1 shows the comparison findings, which were calculated using the mixed beam model (MBM) in [10]. The results of the comparison demonstrate that, as the number of components grows, the result converges to the necessary value and also converges on the published value, as shown in [10]. Because the 8-element mesh size assures accuracy, this mesh will be utilized for the computations in Section 4.

Example 2. The bending behavior of the S-S functionally graded $\mathrm{Al} / \mathrm{ZrO}_{2}$ beam is then compared in this problem. $L / h=16, E_{m}=70 \mathrm{GPa}, E_{c}=200 \mathrm{GPa}$, and $\nu_{c}=v_{m}=0.3$ are the geometrical and material characteristics of the beam, which has a metal ( $\mathrm{Al}$ ) top surface and a ceramic bottom surface $\left(\mathrm{ZrO}_{2}\right)$. The nondimensional maximum deflection of the beam is computed as $\widehat{w}=w_{\max } / 5 q_{0} L^{4} / 384 E_{A l} I$ (with $I=b h^{3} / 12$ ) when the load is evenly distributed. Table 2 lists the nondimensional maximum deflections produced from this research using the Ritz technique [31], where incremental mesh size is used in this case. The 10-element mesh size ensures the requisite precision, as can be observed by the readers. As a result, this mesh will be used in the following investigations.

Example 3. Finally, the findings of nondimensional maximum deflections of the FGM beam resting on the Winkler-Pasternak two-parameter elastic foundation are shown in this case. The length $\mathrm{L}$, breadth $b$, thickness $h$, and $\bar{K}_{w}=$ $K_{1} L^{4} / E I$ and $\bar{K}_{s}=K_{2} L^{2} / E I$ (with $I=b h^{3} / 12$ ) are the geometrical and material characteristics of the beam. A evenly distributed load, $q_{0}$, is applied to the beam. Tables 3 and 4 show nondimensional maximum deflections $\bar{w}=E I$ $/ P_{0} L^{4} w_{\max }$, the differential quadrature method (DQM) [32], and precise solutions $[32,33]$.

\section{Discussion and Numerical Results}

The numerical results of the static bending of the rotating FGM porous beam resting on the two-parameter elastic foundation, with the initial geometrical imperfection taken into account, are presented in this section. The beam is composed of ceramic ( $\mathrm{ZrO} 2)$ and metal (Al), with material characteristics $E_{m}=70 \mathrm{GPa}, E_{c}=200 \mathrm{GPa}, \nu_{c}=v_{m}=0.3$, $\rho_{c}=3960 \mathrm{~kg} / \mathrm{m}^{3}$, and $\rho_{m}=2702 \mathrm{~kg} / \mathrm{m}^{3}$, respectively, with the bottom surface being metal $(\mathrm{Al})$ and the top surface being ceramic $(\mathrm{ZrO} 2)$, and the porosity aspect ratio being $\alpha$. The length of the beam is $L$, and the thickness is $h=L / 10$. Conditions along the border in this section, S-S and C-C, are explored. The beam imperfection is $w_{\text {imp }}(x)=R_{0} \sin (\pi x)$, where $R_{0}$ is the amplitude of the defect and $m=R_{0} / L=0.001$ is the imperfection ratio.

The FGM beam's nondimensional maximum deflection and other characteristics are determined as follows: 
TABLe 1: Nondimensional maximum deflections $\bar{w}$ of the FGM porous beam under uniformly distributed load, S-S, $L / h=5$ and $n=2$.

\begin{tabular}{lccccc}
\hline$\alpha$ & & \multicolumn{3}{c}{ This work } \\
& 6 elements & 8 elements & 10 elements & 12 elements & 14 elements \\
\hline 0 & 5.1701 & 5.1701 & 5.1701 & 5.1701 & 5.1701 \\
0.1 & 5.7959 & 5.7959 & 5.7958 & 5.7958 & 5.7958 \\
0.2 & 6.6146 & 6.6146 & 6.6146 & 6.6146 & 6.6146 \\
\hline
\end{tabular}

TABle 2: Comparative nondimensional maximum deflections $\widehat{w}$ of the FGM beam under uniformly distributed load, S-S, $L / h=16$.

\begin{tabular}{lccccc}
\hline$N$ & This work & Ritz method [31] & $N$ & This work & Ritz method [31] \\
\hline 0 & 1.0093 & 1.00975 & 1 & 0.5662 & 0.56699 \\
0.2 & 0.7561 & 0.75737 & 2 & 0.5073 & 0.50780 \\
0.5 & 0.6391 & 0.64065 & 5 & 0.4441 & 0.44442 \\
\hline
\end{tabular}

TABLE 3: Comparative nondimensional maximum deflections $\bar{w}=E I / P_{0} L^{4} w_{\max }$ of the FGM beam under uniformly distributed load resting on the Winkler-Pasternak two-parameter elastic foundation, S-S.

\begin{tabular}{|c|c|c|c|c|c|}
\hline \multicolumn{2}{|c|}{ Foundation parameters } & \multicolumn{4}{|c|}{$L / h=120$} \\
\hline \multirow[t]{2}{*}{$\bar{K}_{w}$} & $\bar{K}_{s}$ & DQM [32] & Exact [32] & Exact [33] & This work \\
\hline & 0 & 1.302290 & 1.302290 & 1.3033 & 1.301692 \\
\hline \multirow[t]{3}{*}{0} & 10 & 0.644827 & 0.644827 & 0.6457 & 0.644679 \\
\hline & 25 & 0.366111 & 0.366111 & 0.3671 & 0.366063 \\
\hline & 0 & 1.180567 & 1.180567 & 1.1814 & 1.180075 \\
\hline \multirow[t]{3}{*}{10} & 10 & 0.613325 & 0.613326 & 0.6141 & 0.613192 \\
\hline & 25 & 0.355668 & 0.355668 & 0.3566 & 0.355622 \\
\hline & 0 & 0.640074 & 0.640074 & 0.6403 & 0.639927 \\
\hline \multirow[t]{2}{*}{100} & 10 & 0.425582 & 0.425582 & 0.4261 & 0.425517 \\
\hline & 25 & 0.282846 & 0.282846 & 0.2836 & 0.282817 \\
\hline
\end{tabular}

TABLE 4: Comparative nondimensional maximum deflections $\bar{w}=E I / P_{0} L^{4} w_{\max }$ of the FGM beam under uniformly distributed load resting on the Winkler-Pasternak two-parameter elastic foundation, C-C.

\begin{tabular}{lcccc}
\hline Foundation parameters & \multicolumn{3}{c}{$L / h=120$} \\
\hline $\bar{K}_{w}$ & $\bar{K}_{s}$ & DQM [32] & Exact [33] & 0.2616 \\
0 & 0 & 0.26064 & 0.2095 & 0.26033 \\
& 10 & 0.20862 & 0.1617 & 0.20840 \\
& 25 & 0.16081 & 0.2565 & 0.16066 \\
10 & 0 & 0.25547 & 0.2062 & 0.25518 \\
& 10 & 0.20528 & 0.1597 & 0.20507 \\
& 25 & 0.15880 & 0.2174 & 0.216865 \\
100 & 0 & 0.21670 & 0.1800 & 0.17919 \\
& 10 & 0.17935 & 0.1435 & 0.14261 \\
\hline
\end{tabular}

$$
\begin{aligned}
w * & =100 \frac{E_{m} h_{0}^{3}}{P_{0} L^{4}} w_{\max }, \\
K_{w}^{*} & =\frac{k_{w} L^{4}}{D_{0}} \\
K_{s}^{*} & =\frac{k_{s} L^{2}}{D_{0}} \\
\omega & =L^{2} \phi \sqrt{\frac{12 \rho_{m}}{E_{m} h^{2}}}
\end{aligned}
$$

$$
\begin{aligned}
D_{0} & =\frac{E_{m} h^{3}}{12}, \\
N^{*} & =\frac{N_{0} L^{2}}{\pi^{2} E_{m} I_{0}}, \\
I_{0} & =\frac{h^{3}}{12} .
\end{aligned}
$$

4.1. Influence of Rotational Speed. The influence of rotational speed on the structure's maximum deflection will be discussed in this section. Consider a beam with with $L / h=10$ 


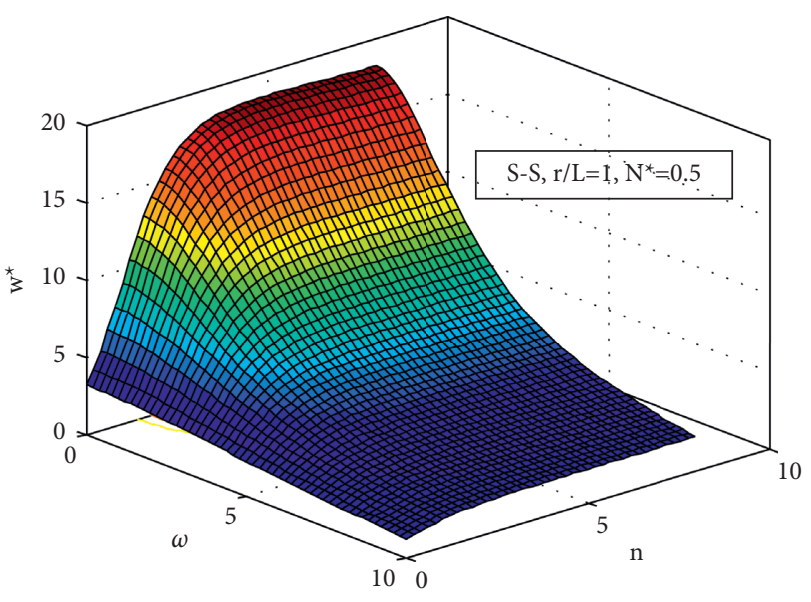

(a)

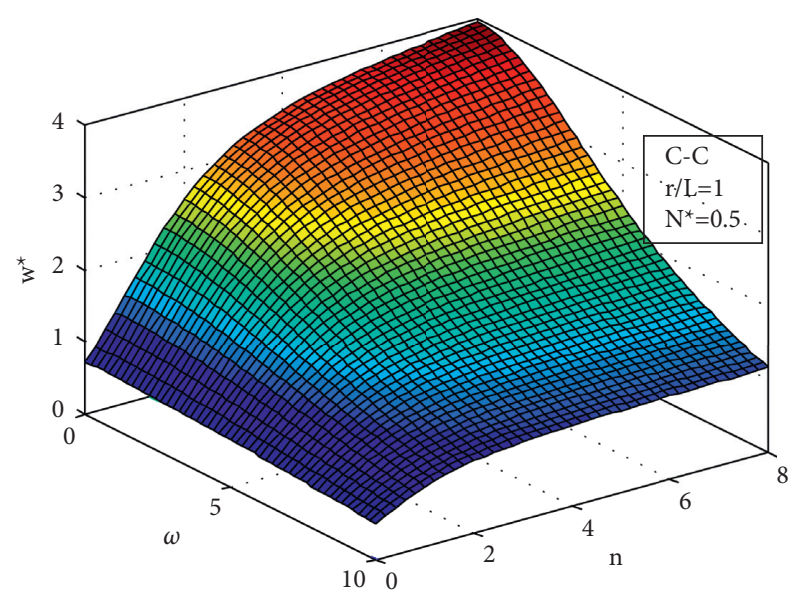

(b)

Figure 2: The nondimensional maximum deflection of the beam as a function of rotational speed, $K_{w}^{*}=20, K_{s}^{*}=5$, and $\alpha=0.2$. (a) S-S. (b) C-C.

and $\alpha=0.2$, geometrical imperfection coefficient $m=0.001$, $K_{w}^{*}=20$ and $K_{s}^{*}=5$, and the distance ratio $r / L=1$. The rotational speed $\omega$ rises from 0 to 10 , and the volume fraction exponent $n$ rises from 0 to 8 . Figure 2 shows the dependency of the maximum deflection on the rotational speed and the volume fraction exponent, while Figure 3 shows the dependence on the rotational speed and the distance ratio $r / L$. Figures 4-5 show the deflection line of the beam as a function of the distance ratio $r / L$ and the rotating speed. As a result of the numerical data, the following conclusions may be drawn:

(i) As the rotating speed increases, the maximum deflection of the beam reduces, demonstrating that the centrifugal force changes the beam stiffness. This also implies that the rotational speed improves the beam's bearing capacity. When the speed parameter of the beam is between 0 and 20 , the maximum deflection of the beam falls rapidly; when the value is more than 20 , the maximum deflection decreases gently.

(ii) As the rotating speed and the $r / L$ ratio rise, the maximum deflection value lowers as well. At the same time, the maximum deflection position shifts to the right in relation to the midbeam position, which is entirely different from beams with no rotating movement. This demonstrates that the rotation speed and $r / L$ ratio have an impact on the form of the deflection curve of the beam.

(iii) As the volume exponent $n$ is increased, the amount of metal material composition in the beam increases, reducing the stiffness of the beam and lowering the maximum deflection of the beam.

4.2. Effect of the Axial Compressive Load. The impact of the axial compressive load on the static bending response of porous FG beams is investigated in this section. The compressive load $N^{*}$ is chosen for this purpose, with a value ranging from 0 to 1 . Figure 6 shows the greatest deflections of the FG beam based on the value of $N^{*}$ related to the various rotating speeds. The maximum deflection of the beam rises as the $N^{*}$ increases; however, the rotating speed has a major impact on the increase of the maximum deflections of the beam. When the compression force $N^{*}$ rises for a small rotation speed, the maximum deflection of the beam increases substantially, but when $N^{*}$ increases for a higher rotation speed, the maximum deflection of the beam increases just little. Figure 7 shows that, as the compressive load $N^{*}$ increases, the maximum deflection of the porous FG beam increases, but the increase in maximum deflection is not the same when the material volume exponent is different; with $n$ having a larger value, the proportion of metal in the beams is increased and the beam is softer, so the compressive load influence is most clearly manifested.

4.3. Effect of the Porosity Volume Fraction. The coefficient $\alpha$ takes values from 0 to 0.5 to clearly illustrate the impact of the porosity volume fraction on the static bending response of FG beams, and the results of calculating the maximum deflection of the FG beams depend on $\alpha$ and the rotational speed are presented in Figure 8 . These results show that when the porosity volume fraction increases, the beam gets softer, resulting in a higher maximum deflection. The increase in deflection, however, is most noticeable when the rotational speed of the beam is low due to the impact of centrifugal force. Figure 9 depicts the deflection curve of this beam as a function of varied porosity volume fraction values. The porosity volume fraction increases not only the maximum deflection of the beam but also the position of the maximum deflection, as the reader can see. The greatest deflection tends to deflect to the right, away from the rotation axis of the beam, implying that the coefficient has an impact on the form of the deflection response of FG beams.

4.4. Effect of the Elastic Foundation. Change the elastic foundation parameter $K_{w}^{*}$ to a value between 0 and 100; the coefficient $K_{s}^{*}=5$ remains unchanged. Figure 10 shows the deflection curve for several values of the elastic foundation 


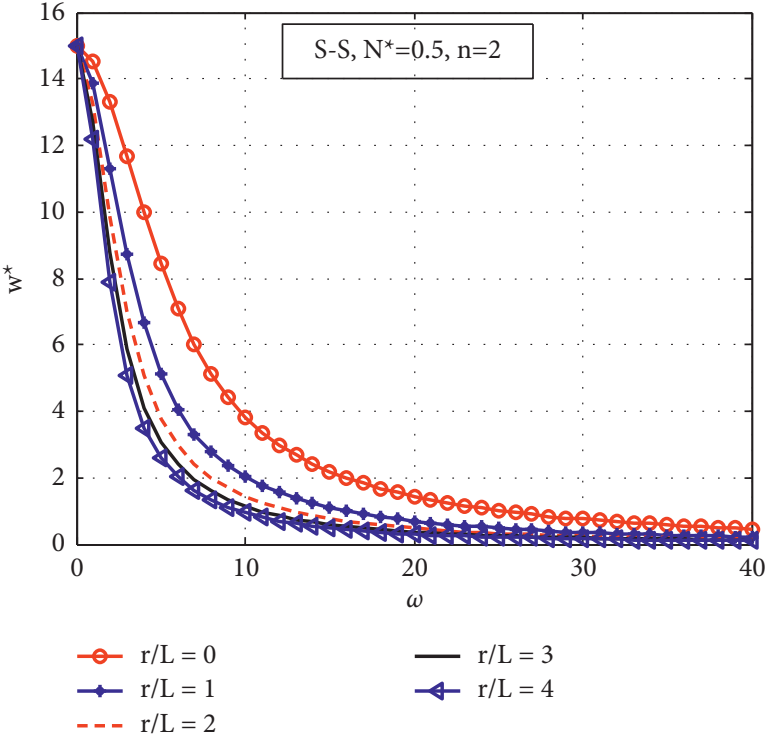

(a)

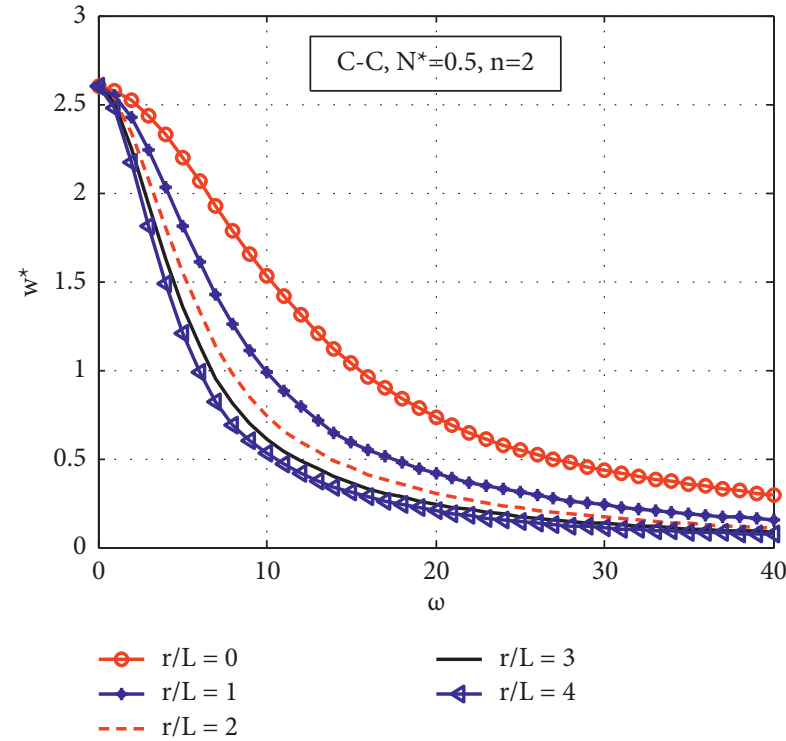

(b)

FIGURE 3: The nondimensional maximum deflection of the FG beam as a function of rotating speed and $(\mathrm{r}) /(\mathrm{L})$ ratio, $K_{w}^{*}=20, K_{s}^{*}=5$, and $\alpha=0.2$. (a) S-S. (b) C-C.

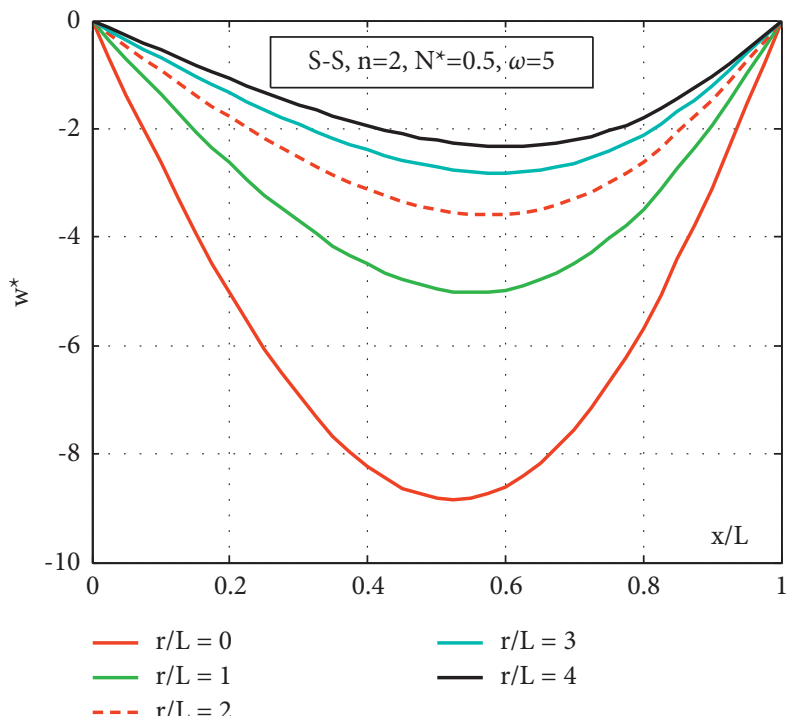

(a)

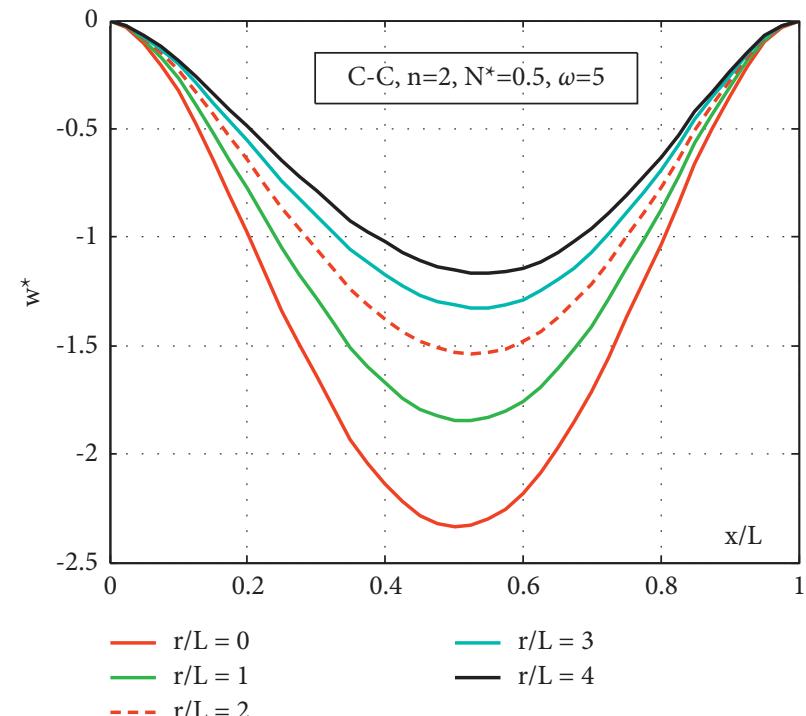

(b)

Figure 4: The deflection line of the FG beam on the (r)/(L) ration, $K_{w}^{*}=20, K_{s}^{*}=5$, and $\alpha=0.2$. (a) S-S. (b) C-C.

parameter $K_{w}^{*}$. The results of this calculation demonstrate that when the elastic foundation's coefficient rises, the maximum deflection of the beam lowers and the position of the maximum deflection of the beam changes as well. This shift, however, is not substantial or obvious, indicating that the elastic foundation coefficient impacts the maximum value of the beam deflection but has minimal effect on the point where the maximum deflection occurs. 


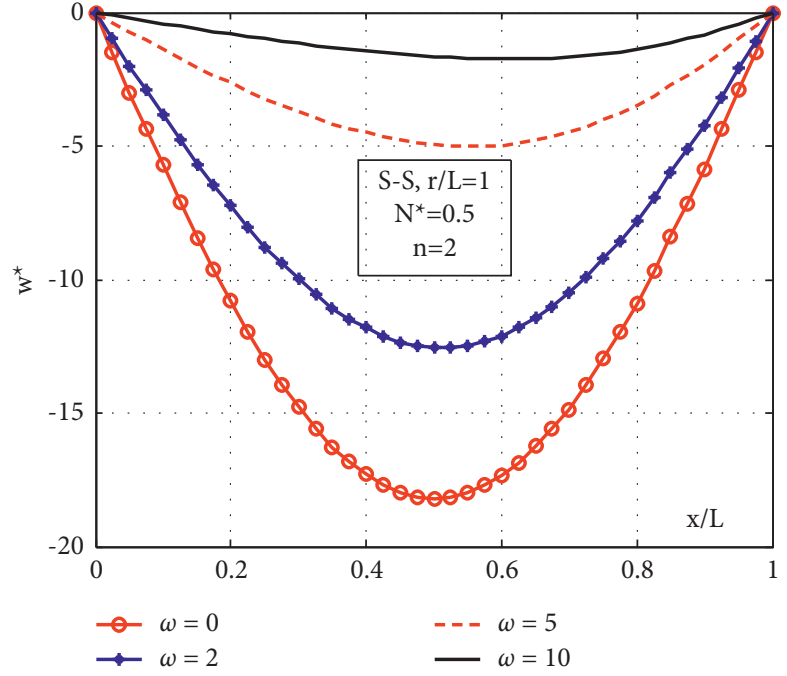

(a)

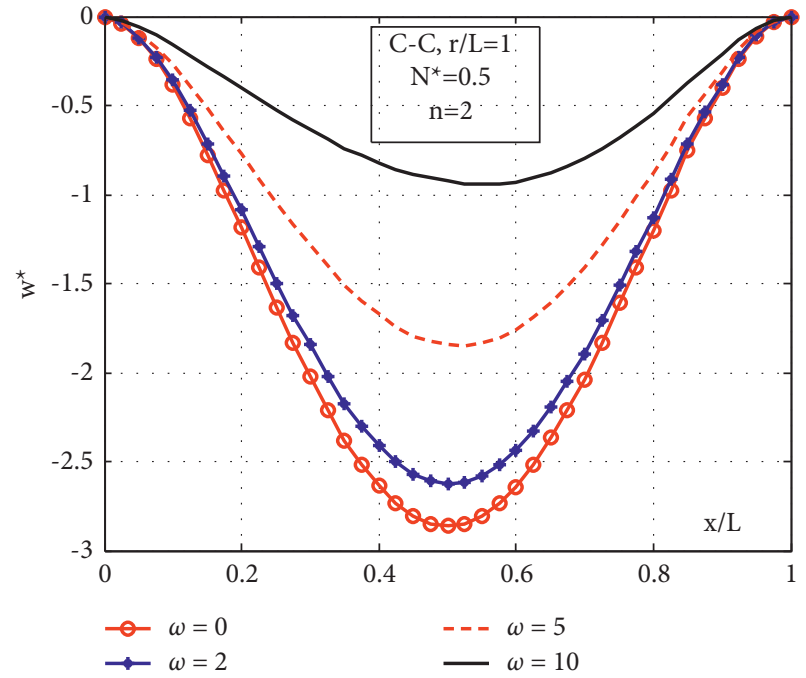

(b)

Figure 5: The FG beam's deflection line as a function of rotating speed, $K_{w}^{*}=20, K_{s}^{*}=5$, and $\alpha=0.2$. (a) S-S. (b) C-C.

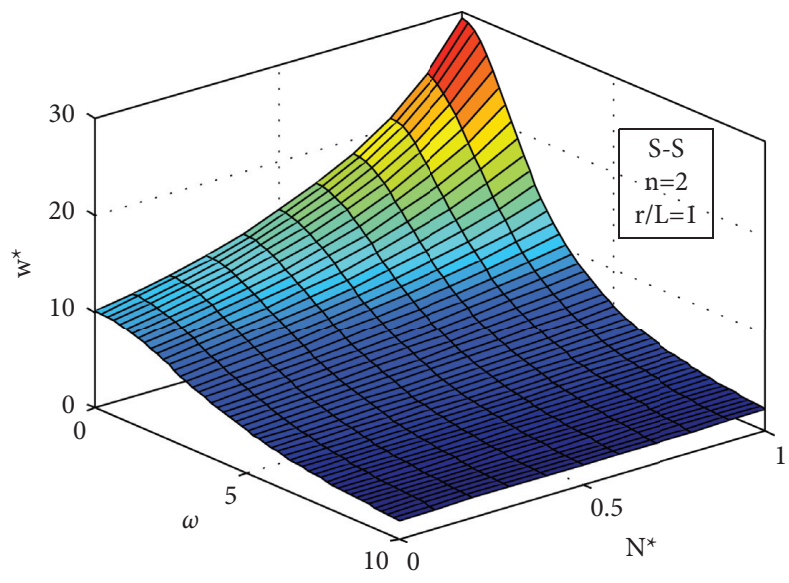

(a)

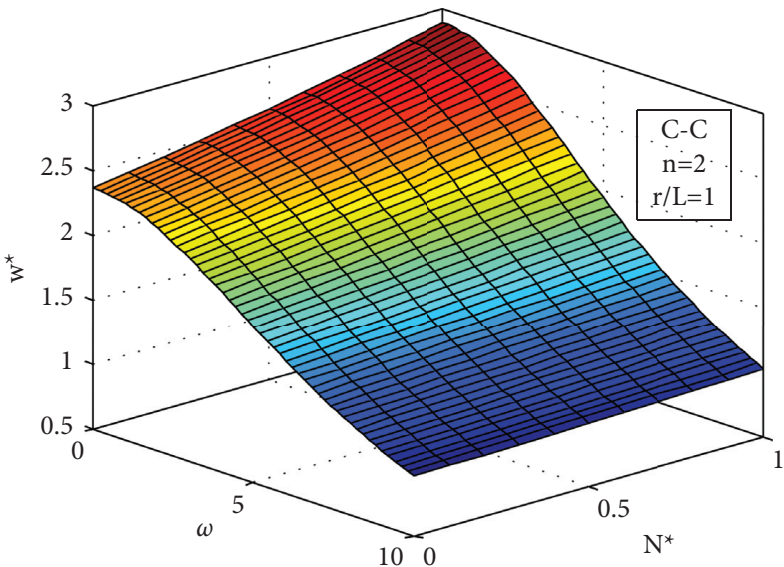

(b)

FIgURE 6: The nondimensional maximum deflection of the FG beam is influenced by the axial compressive load and rotating speed, $K_{w}^{*}=20$, $K_{s}^{*}=5$, and $\alpha=0.2$. (a) S-S. (b) C-C. 


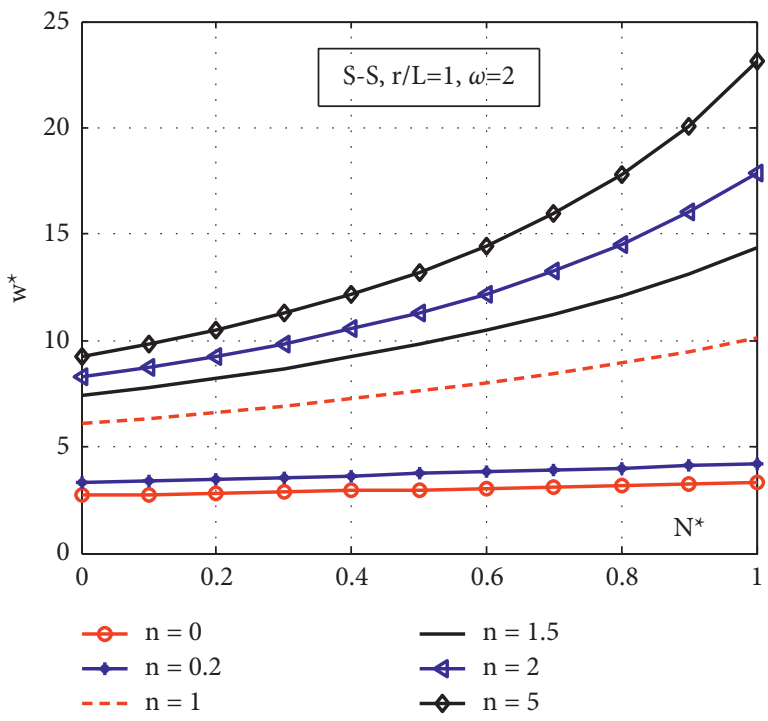

(a)

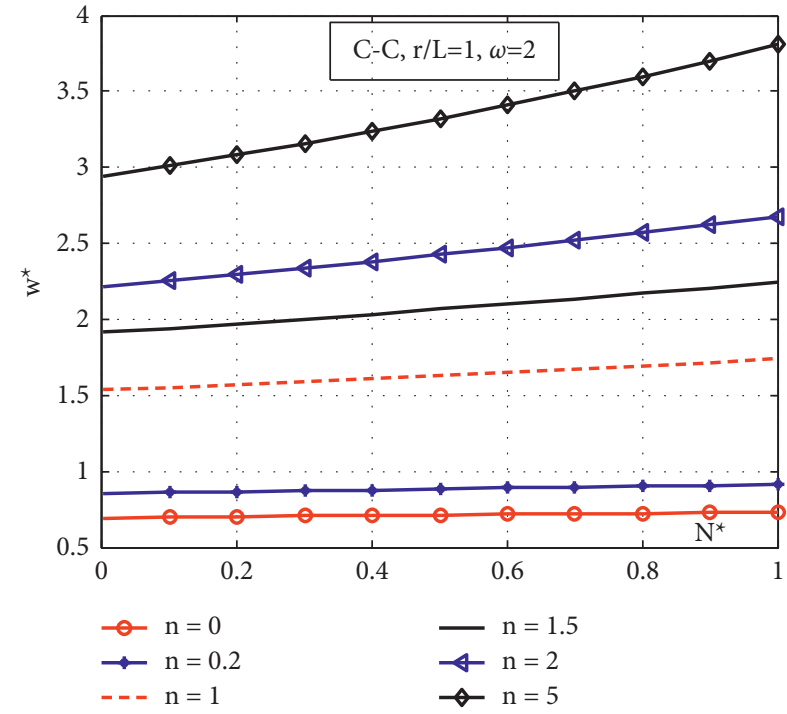

(b)

FIGURE 7: The relationship between the axial compressive stress and the volume fraction exponent $(n)$ and the nondimensional maximum deflection of the FG beam, $K_{w}^{*}=20, K_{s}^{*}=5$, and $\alpha=0.2$. (a) S-S. (b) C-C.

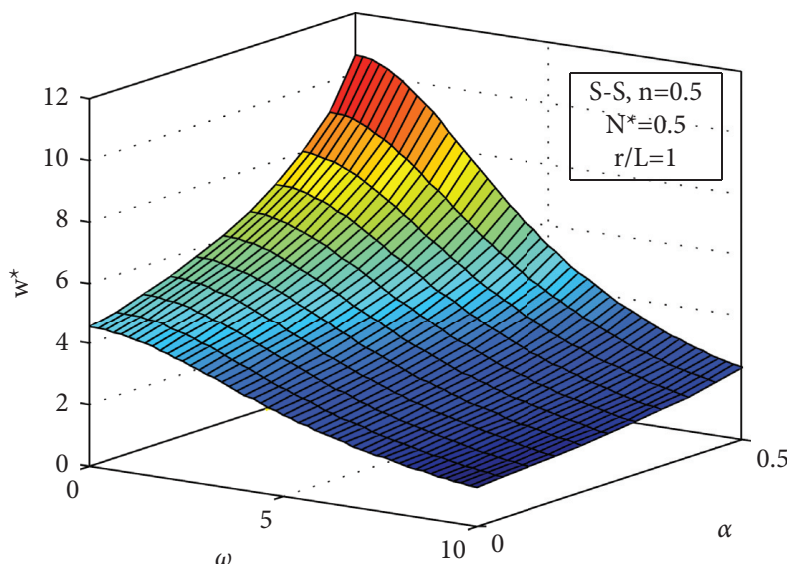

(a)

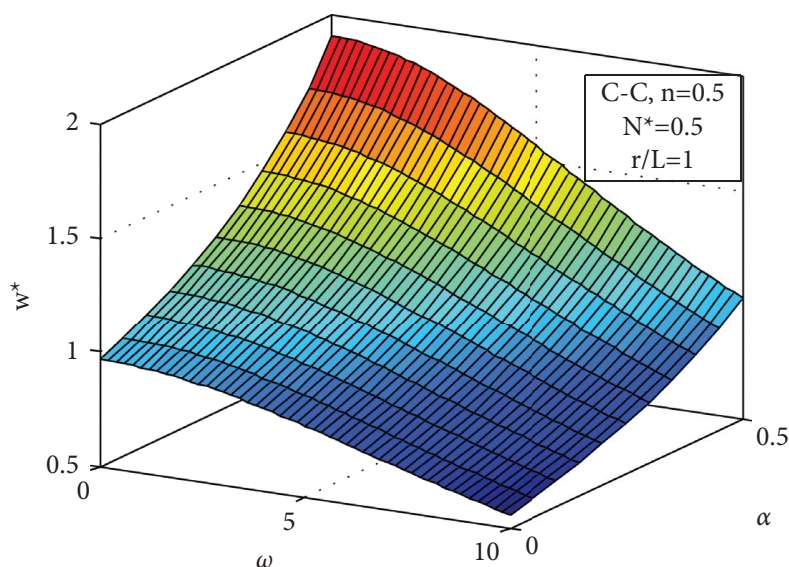

(b)

FIGURE 8: The nondimensional maximum deflection of the FG beam as a function of porosity volume fraction $\alpha$ and rotating speed, $K_{w}^{*}=20$ and $K_{s}^{*}=5$. (a) S-S. (b) C-C. 


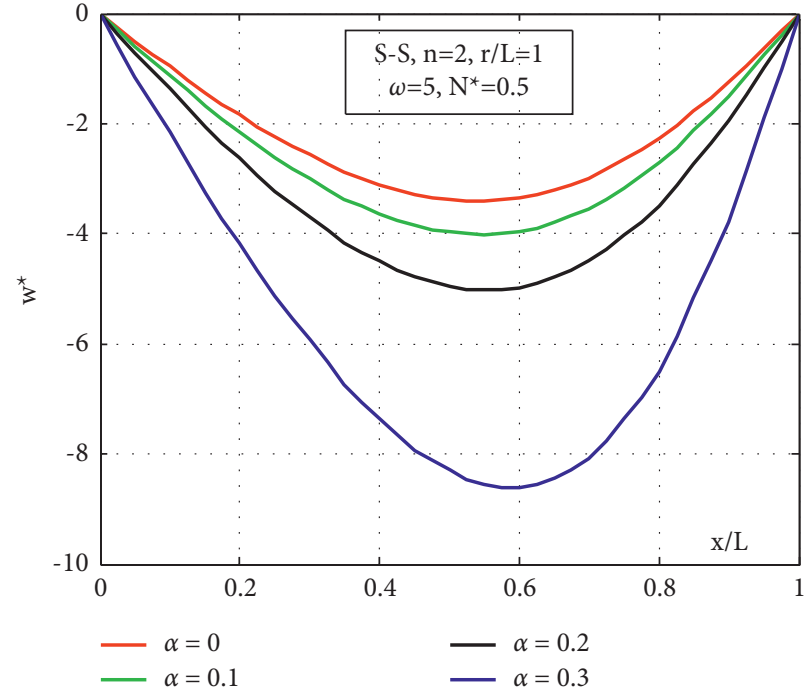

(a)

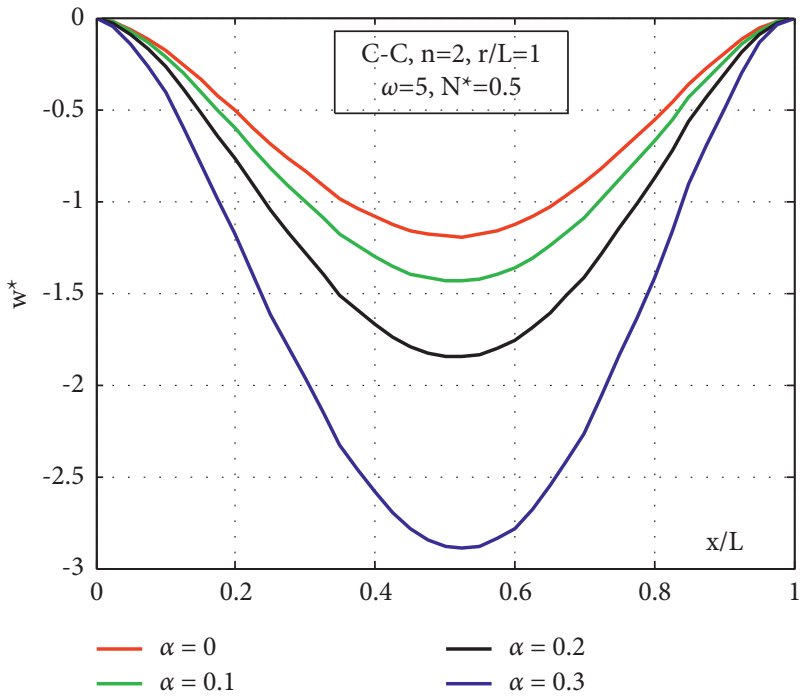

(b)

Figure 9: The relationship between the porosity volume fraction $\alpha$ and the FG beam's deflection curve, $K_{w}^{*}=20$ and $K_{s}^{*}=5$. (a) S-S. (b) C-C.

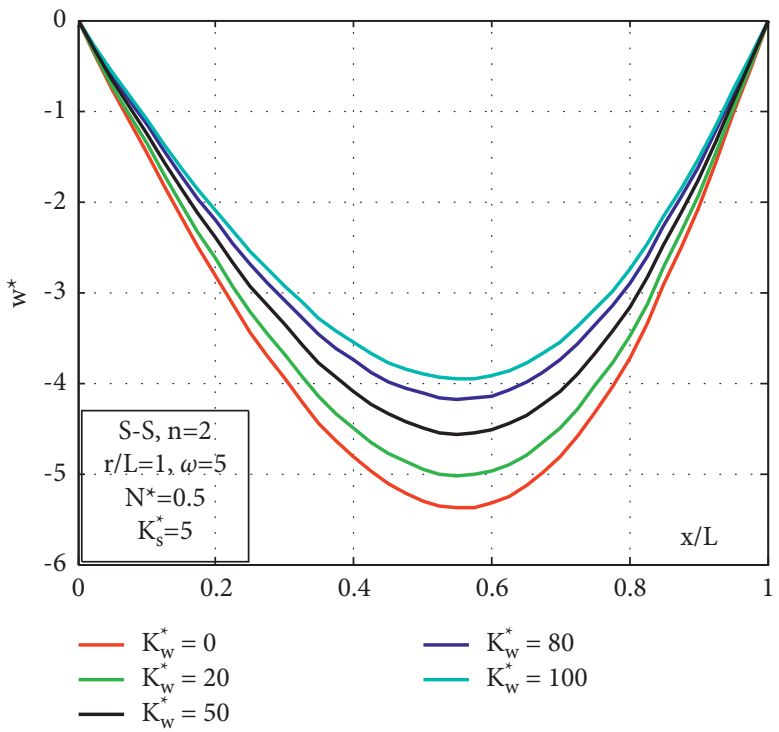

(a)

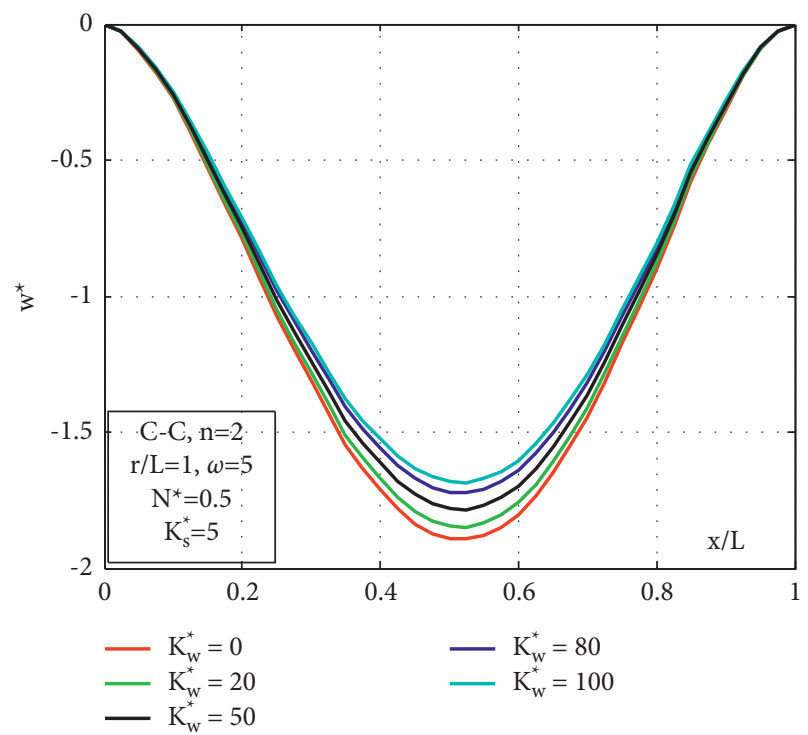

(b)

FIgURE 10: The dependence of the FG beam's deflection curve on the elastic foundation parameter, $K_{w}^{*}$ and $K_{s}^{*}=5$. (a) S-S. (b) C-C.

\section{Conclusions}

The static bending of a porous FG beam resting on a twoparameter elastic foundation is initially simulated using the third-order shear deformation theory and finite element technique, in which the beam rotates around one fixed axis and an initial geometrical defect is taken into consideration. Some novel points may be drawn from the numerical results as follows:

(i) As the rotating movement's speed increases, the presence of centrifugal force lowers the beam's maximum deflection. However, when the rotational speed is between 0 and 20, the change in the maximum deflection of the beam is most noticeable, and when it is more than 20 , the influence of this speed on the deflection response is minimal.

(ii) The maximum deflection of the beam increases as the axial compressive load $N^{*}$ increases, although the impact of $N^{*}$ on the maximum deflection of the beam is most clearly exhibited when the speed of rotation is modest.

(iii) The maximum deflection reduces as the elastic foundation coefficient is increased and the porosity volume percentage is reduced. When the values of 
these parameters are modified, the deflection curve is likewise adjusted.

\section{Data Availability}

The data used to support the findings of this study are included in the article.

\section{Conflicts of Interest}

All authors declare that there are no conflicts of interest regarding the publication of this paper.

\section{Acknowledgments}

This work was supported by the University of transport technology Foundation for Science and Technology Development (Grant no. 1139/QD-DHCNGTVT).

\section{References}

[1] S. D. Akbaş, "Nonlinear static analysis of functionally graded porous beams under thermal effect," Coupled Syst. Mech.vol. 6, pp. 399-415, 2017.

[2] A. Mojahedin, M. Jabbari, and T. Rabczuk, "Thermoelastic analysis of functionally graded porous beam," Journal of Thermal Stresses, vol. 41, no. 8, pp. 937-950, 2018.

[3] M. A. Eltaher, N. Fouda, T. El-Midany, and A. M. Sadoun, "Modified porosity model in analysis of functionally graded porous nanobeams," Journal of the Brazilian Society of Mechanical Sciences and Engineering, vol. 40, no. 3, 2018.

[4] M. Hamed, A. Sadoun, and M. A. Eltaher, "Effects of porosity models on static behavior of size dependent functionally graded beam," Structural Engineering \& Mechanics, vol. 71, pp. 89-98, 2019.

[5] B. Fahsi, R. B. Bouiadjra, A. Mahmoudi, S. Benyoucef, and A. Tounsi, "Assessing the effects of porosity on the bending, buckling, and vibrations of functionally graded beams resting on an elastic foundation by using a new refined quasi-3D theory," Mechanics of Composite Materials, vol. 55, no. 2, pp. 219-230, 2019.

[6] B. Anirudh, M. Ganapathi, C. Anant, and O. Polit, "A comprehensive analysis of porous graphene-reinforced curved beams by finite element approach using higher-order structural theory: bending, vibration and buckling," Composite Structures, vol. 222, Article ID 110899, 2019.

[7] O. Polit, C. Anant, B. Anirudh, and M. Ganapathi, "Functionally graded graphene reinforced porous nanocomposite curved beams: bending and elastic stability using a higherorder model with thickness stretch effect," Composites Part B: Engineering, vol. 166, pp. 310-327, 2019.

[8] P. K. Masjedi, A. Maheri, and P. M. Weaver, "Large deflection of functionally graded porous beams based on a geometrically exact theory with a fully intrinsic formulation," Applied Mathematical Modelling, vol. 76, pp. 938-957, 2019.

[9] Y. Gao, W.. Xiao, Nonlinear bending of functionally graded porous nanobeam subjected to multiple physical load based on nonlocal strain gradient theory," Steel and Composite Structures, vol. 31, no. 5, pp. 469-488, 2019.

[10] S. Zghal, D. Ataoui, and F. Dammak, "Static bending analysis of beams made of functionally graded porous materials," Mechanics Based Design of Structures and Machines, pp. 1-18, 2020.
[11] M. A. Hamed, R. M. Abo-bakr, S. A. Mohamed, and M. A. Eltaher, "Influence of axial load function and optimization on static stability of sandwich functionally graded beams with porous core," Engineering with Computers, vol. 36, no. 4, pp. 1929-1946, 2020.

[12] Z. Nan, Z. Xie, Z. Shijie, and C. Dejin, "Size-dependent static bending and free vibration analysis of porous functionally graded piezoelectric nanobeams," Smart Materials and Structures, vol. 29, no. 4, Article ID 045025, 2020.

[13] S. Enayat, M. Hashemian, D. Toghraie, and E. Jaberzadeh, "Bending, buckling and vibration analyses of FG porous nanobeams resting on Pasternak foundation incorporating surface effects," ZAMM - Journal of Applied Mathematics and Mechanics/Zeitschrift für Angewandte Mathematik und Mechanik, 2020.

[14] X. Zhao, S. Zheng, and Z. Li, "Effects of porosity and flexoelectricity on static bending and free vibration of AFG piezoelectric nanobeams," Thin-Walled Structures, vol. 151, Article ID 106754, 2020.

[15] M. R. Pajand, N. R. Safaei, and A. R. Masoodi, "An efficient curved beam element for thermo-mechanical nonlinear analysis of functionally graded porous beams," Structure, vol. 28, pp. 1035-1049, 2020.

[16] A. Karamanli and T. P. Vo, "Bending, vibration, buckling analysis of bi-directional FG porous microbeams with a variable material length scale parameter," Applied Mathematical Modelling, vol. 91, pp. 723-748, 2021.

[17] S. C. Pradhan and T. Murmu, "Application of nonlocal elasticity and DQM in the flapwise bending vibration of a rotating nanocantilever," Phys. E Low-dimensional Syst. Nanostructures, vol. 42, no. 7, pp. 1944-1949, 2010.

[18] L. Li, D. G. Zhang, and W. D. Zhu, "Free vibration analysis of a rotating hub-functionally graded material beam system with the dynamic stiffening effect," Journal of Sound and Vibration, vol. 333, no. 5, pp. 1526-1541, 2014.

[19] D. Das, "Free vibration and buckling analyses of geometrically non-linear and shear-deformable FGM beam fixed to the inside of a rotating rim," Composite Structures, vol. 179, pp. 628-645, 2017.

[20] D. Chen, K. Feng, and S. Zheng, "Flapwise vibration analysis of rotating composite laminated Timoshenko microbeams with geometric imperfection based on a re-modified couple stress theory and isogeometric analysis," European Journal of Mechanics - A: Solids, vol. 76, pp. 25-35, 2019.

[21] N. V. Dang, "A third-order shear deformation theory for bending behaviors of rotating FGM beams resting on elastic foundation with geometrical imperfections in thermal environments," Mathematical Problems in Engineering, vol. 2021, pp. 1-19, 2021.

[22] T. Q. Bui, "On the high temperature mechanical behaviors analysis of heated functionally graded plates using FEM and a new third-order shear deformation plate theory," Composites Part B: Engineering, vol. 92, pp. 218-241, 2016.

[23] H.-N. Nguyen, T. C. Tan, D. T. Luat, V.-D. Phan, D. V. Thom, and P. V. Minh, "Research on the buckling behavior of functionally graded plates with stiffeners based on the thirdorder shear deformation theory," Materials, vol. 12, no. 8, p. 1262, 2019.

[24] N. V. Hoai, D. H. Doan, N. M. Khoa, T. V. Do, and H. T. Tran, "Phase-field buckling analysis of cracked stiffened functionally graded plates," Composite Structures, vol. 217, pp. 50-59, 2019.

[25] T. Yu, T. Q. Bui, S. Yin et al., "On the thermal buckling analysis of functionally graded plates with internal defects 
using extended isogeometric analysis," Composite Structures, vol. 136, pp. 684-695, 2016.

[26] P. T. Dat, D. V. Thom, and D. T. Luat, "Free vibration of functionally graded sandwich plates with stiffeners based on the third-order shear deformation theory," Vietnam Journal of Mathematics, vol. 38, no. 2, pp. 103-122, 2016.

[27] N. D. Duc, T. D. Trinh, T. V. Do, and D. H. Doan, "On the buckling behavior of multi-cracked FGM plates,"pp. 29-45, Singapore, February 2018.

[28] T. Van Do, D. H. Doan, N. D. Duc, and T. Q. Bui, "Phase-field thermal buckling analysis for cracked functionally graded composite plates considering neutral surface," Composite Structures, vol. 182, pp. 542-548, 2017.

[29] J. N. Reddy, "Analysis of functionally graded plates," International Journal for Numerical Methods in Engineering, vol. 47 , no. 1-3, pp. 663-684, 2000.

[30] S. P. Timoshenko and J. M. Gere, Theory of Elastic Stability, Dover Publications, New York, NY, USA, 1989.

[31] M. Simsek, "Static analysis of a functionally graded beam under a uniformly distributed load by Ritz method," International Journal of Engineering and Applied Sciences, vol. 1, 2009.

[32] W. Q. Chen, C. F. Lü, and Z. G. Bian, "A mixed method for bending and free vibration of beams resting on a Pasternak elastic foundation," Applied Mathematical Modelling, vol. 28, no. 10, pp. 877-890, 2004.

[33] C. M. Wang, K. Y. Lam, and X. Q. He, "Exact solutions for Timoshenko beams on elastic foundations using green's Functions*," Mechanics of Structures and Machines, vol. 26, no. 1, pp. 101-113, 1998. 